\title{
Progress in multipass tandem Fabry-Perot interferometry: I. A fully automated, easy to use, self-aligning spectrometer with increased stability and flexibility
}

\author{
Burkard Hillebrands \\ Fachbereich Physik und Schwerpunkt Materialwissenschaften, Universität Kaiserslautern, Erwin- \\ Schrödinger-Straße 56, 67663 Kaiserslautern, Germany
}

(Received 25 August 1998; accepted for publication 8 November 1998)

\begin{abstract}
A computer control for a Sandercock-type multipath tandem Fabry-Perot interferometer is described, which offers many advantages over conventionally used analog control: The range of stability is increased due to active control of the laser light intensity and the mirror dither amplitude. The alignment is fully automated enabling start of a measurement within a minute after start of align, including optionally finding the optimum focus on the sample. The software control enables a programmable series of measurements with control of, e.g., the position and rotation of the sample, the angle of light incidence, the sample temperature, or the strength and direction of an applied magnetic field. Built-in fitting routines allow for a precise determination of frequency positions of excitation peaks combined with increased frequency accuracy due to a correction of a residual nonlinearity of the mirror stage drive. (c) 1999 American Institute of Physics.
\end{abstract}

[S0034-6748(99)03202-5]

\section{INTRODUCTION}

For over a decade high-contrast tandem Fabry-Perot interferometers have been installed and used to investigate weak light signals scattered inelastically from surface and thin-film phonons and from spin wave excitations. The central, frequency selecting element of the setup is an etalon consisting of two highly reflective, parallel mirrors. Light is transmitted through the etalon if the mirror spacing is a multiple of half its wavelength, $\lambda$.

In conventional interferometry using one etalon the analysis of inelastic excitations is disturbed by the ambiguous assignment of measured frequency shifts to the appropriate transmission order, since the transmission is periodic in $\lambda / 2$ in the mirror plate spacing. These ambiguities are avoided in the tandem etalon arrangement invented by Sandercock ${ }^{1-5}$ (see also Ref. 6 for a different approach). Multipass operation allows for a high contrast of better than $10^{10}$ enabling detection of elastic and magnetic surface excitations even in metallic systems with a sensitivity down to the monolayer regime, as shown for the case of spin waves in Co films. ${ }^{7}$ However, the requirements on the alignment of the optical setup are extremely high. They can only be maintained over the time of a measurement by an active stabilization scheme. Sandercock reported first the successful installation of an actively stabilized tandem Fabry-Perot interferometer. ${ }^{1}$

To achieve long-term stability of the etalon mirror alignment, several measures have been proposed and realized: First, a very good isolation from ground vibrations is needed. This is obtained by an optic table and/or, as recently introduced, by an actively stabilized table as the mount of the etalon mirrors. ${ }^{8}$ Second, thermal and acoustic isolation of the etalon mirrors can be obtained by a box surrounding the interferometer setup and coated with sound isolation and thermal isolation material. Third, and most crucial, an active stabilization mechanism for maintaining parallelity and correlation between the etalon mirror pairs is of key importance. For the latter, several solutions using analog circuits, ${ }^{9-11}$ digital hardware, ${ }^{5,12}$ or a personal computer ${ }^{13,14}$ have been proposed.

In discussions about the applicability of the Brillouin light scattering technique two major obstacles are often named, which hamper a successful experiment: First, due to the rather complicated optical setup and the high requirements on the optical alignment, it needs a very experienced, thoroughly trained person to run the experiment. Second, the system must be kept in the aligned state over the time of measurement, i.e., the system must be designed in such a stable way, that destabilization remains a rare event. It is often said, that the required full attendance of the operator to the progress of the measurement is too time consuming a task.

Our aim was to develop an easy to use, automated Brillouin light scattering spectrometer built on the Sandercock setup, which allows for full unattended operation after starting a programmed sequence of measurements. We report on the development of a computer based controller, which, due to the flexibility of a software solution, allows (i) to align the system automatically, (ii) to maintain stabilization during measurements with fallback to autoalign in case of an event of destabilization, (iii) to collect and handle the data, and (iv) to control all peripheral devices. Due to the intrinsic versatility of a software solution the everyday stability of the system is superior to existing hardware solutions with the additional advantage of easy control and handling of all signal and data flow. Due to the simple interface to the user, the entire Brillouin light scattering setup can be operated by any researcher after only a few days training in the operation. 


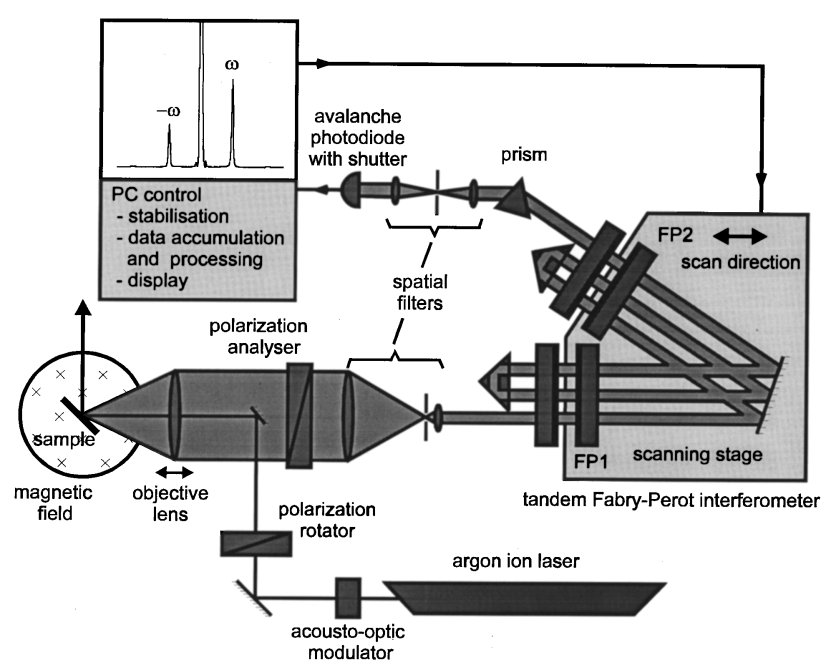

FIG. 1. Schematic setup of the spectrometer. The optical beam pass is shown for the $(3+3)$ pass geometry. Not shown is the slide with glass blocks and half-reflecting mirrors for switching into the reflection mode for alignment of the system. Also not shown is the system for positioning the sample.

Our solution builds on a computer controlled tandem Fabry-Perot interferometer designed and built in 1984-86 by Bell, Zanoni, and Stegeman at the Optical Sciences Center at the University of Arizona. ${ }^{14}$ They developed software, written in Forth, which allowed for active stabilization of the setup and for data accumulation. For the latter a conventional multichannel analyzer was used and interfaced with the PC. Their setup, although state of the art at the time of construction, did not exhibit the robustness and flexibility of our design, in particular since their solution did not allow for automated series of measurements, for autoalignment and autofocus, and for control over peripheral devices.

\section{DESCRIPTION OF OUR SETUP AND PRINCIPLE OF OPERATION}

We first outline shortly the layout and the principle of operation of our setup. For a full discussion of the operation the reader might consult the literature. ${ }^{1-4,9-11,15}$

\section{A. Description of our setup}

The setup used in our laboratory is schematically shown in Fig. 1. Light of a frequency stabilized argon ion laser $(\lambda=514.5 \mathrm{~nm}, \Delta v=20 \mathrm{MHz})$ is focused onto the sample with an objective lens (focal length $50 \mathrm{~mm}$ ). The lens is mounted on a motor driven slide and can be adjusted under computer control. The sample is mounted on a motor driven stage. Under computer control the position of the laser focus on the sample, the angle of light incidence as well as the in-plane direction of wave vector transfer can be set. An optional magnetic field, needed for spin wave investigations, is applied parallel to the sample surface and perpendicular to the scattering plane.

An acousto-optic modulator (AOM) allows to vary the light intensity under computer control. The polarization rotator is used to adjust the polarization direction of the linearly polarized light.
We usually use the setup in backscattering geometry as displayed in Fig. 1, but any other scattering geometry can be easily realized. For example, for investigations of the spin wave intensity distribution in yttrium-iron-garnet films we use the forward scattering geometry. ${ }^{16-18}$

In backscattering geometry the light scattered from the sample (elastic and inelastic contributions) is collected by the same objective lens and, after optional polarization analysis, it is sent through a spatial filter for suppressing background noise before entering the tandem interferometer. The frequency selected light transmitted by the interferometer is detected by an avalanche photodiode with high quantum efficiency and low dark count rate $(\leqslant 3$ counts/s), after passing through a second spatial filter for additional background suppression. A band pass filter comprising a prism and the second spatial filter serves for suppression of inelastic light from common transmission orders outside the frequency region of interest. A computer processes the photon counts and displays the data.

The light passes in series through two Fabry-Perot etalons FP1 and FP2. One of the two mirrors of both etalons is mounted on a common translation stage which is driven by a piezoelectric crystal (called Z-piezo hereafter). By ramping the voltage $V_{Z}$ applied to the $Z$ piezo the resonance condition for both etalons is varied over the frequency region chosen for the measurement. Using a capacitive distance control of the mirrors in a feedback loop a linear relation between the applied voltage, $V_{Z}$, and the distance between the mirror plates is achieved. ${ }^{19}$

Due to the common mounting of one of the mirrors of each etalon on the translation stage, the change in the spacing of the second etalon is smaller by a factor of $\cos (\theta)$ with $\theta=18.1^{\circ}$ the angle between the optical axes of the two interferometers. Since the transmission of light for a given wavelength $\lambda$ is synchronized only for the preset transmission orders of both etalons, signals from neighboring transmission orders are suppressed (so-called "ghost peaks"), and the inelastic signal represents closely the scattering cross section of the sample. For a detailed discussion of the tandem operation see Ref. 4.

For an experimental realization a flatness of the mirror surfaces of better than $\lambda / 200$ and a parallelity of $\lambda / 100$ of the two mirrors of each etalon are necessary. The latter is achieved by mounting one of the two mirrors of each etalon on three piezoelectric crystals (see also below). ${ }^{19}$ By applying voltages to the piezos the mirrors can be tilted about their $x$ and $y$ axes. We call the corresponding voltages $V_{X 1}$ and $V_{Y 1}$ for the first etalon, FP1, and $V_{X 2}$ and $V_{Y 2}$ for the second etalon, FP2. By applying a constant offset voltage, $V_{d Z}$, to the piezos of FP2 the spacing of the second etalon can be varied by a few wavelengths. This is used for synchronization of both etalons (resonance of both etalons at common frequency).

In order to obtain the high contrast needed to detect the weak inelastic signals, the light is sent through both etalons three times each, using a system of retroreflectors and mirrors as indicated in Fig. 1. The entire system of scanning stage, optic beam path, pin holes, and photon detector is obtained from Ref. 19. 
Modern interferometers are most often setup in the (3 +3 )-pass arrangement. Special measures are taken to protect the detector from overload while scanning through the elastic peak. This is achieved by using the AOM shown in Fig. 1, or by a mirror/shutter system (mirrors and shutters not shown in Fig. 1). In the latter case a small fraction of the laser light (called reference beam) is focused onto the entrance pin hole of the tandem interferometer providing the light for stabilization. Two shutters, one in the light path of the reference beam, the other in the path of the light from the sample and entering the interferometer, control the light flow entering the interferometer. They are switched by the computer. In our setup we can change between both modes of operation.

The AOM has the advantage, that the dynamic range of stabilization is increased due to control of the laser light intensity by varying the AOM input signal. On the other hand, due to the limited dynamic range of the AOM of $<10^{4}$ the suppression of the transmitted elastically scattered light might be insufficient for samples with large elastic scattering contributions. In the latter case the shutter system is used. Using the AOM a direct control of the elastically scattered light is given by monitoring, e.g., the AOM level setting.

The shutter system allows one to maintain stabilization during, e.g., sample positioning since for stabilization the light from the reference beam is used. However, although this might seem an advantage, there is no real need for us to keep the system in an aligned state while working on the sample since the autoalignment is fast and convenient. There are two major disadvantages working with the shutter system. (i). There is no direct control of the elastically scattered light during data accumulation, and (ii) the automatic dither adjustment does not work, since it is closely interconnected with the AOM control (see Sec. III D).

Samples showing an extremely low amount of elastically scattered light are investigated using the shutter system. This is the case, e.g., for spin wave investigations of a sample positioned in an ultrahigh vacuum chamber, since the sample surface is not contaminated with dust and the crossed polarizer/analyzer configuration further suppresses the elastically scattered light.

\section{B. Coarse alignment of the interferometer}

The optical beam path, as developed and distributed by Sandercock, ${ }^{19}$ can be switched between two modes under computer control: The first mode, called "transmission mode" hereafter, is the multipass arrangement described above and shown in Fig. 1, used for taking spectra. The second mode, called "reflection mode," is used for the coarse alignment of the system and it is displayed in Fig. 2. Here the beam pass is as follows: the light impinging on the first etalon, FP1, is fully reflected, if FP1 is not in resonance. Two half-reflecting mirrors direct the back-reflected light onto the second etalon, FP2. The light back-reflected from FP2 goes through the second half-reflecting mirror to the detector. The advantage of this beam arrangement is that it allows us to adjust the parallelism for both etalons independently as follows: The reflection coefficient of each etalon is close to one, if the etalon is not in resonance. By sweeping

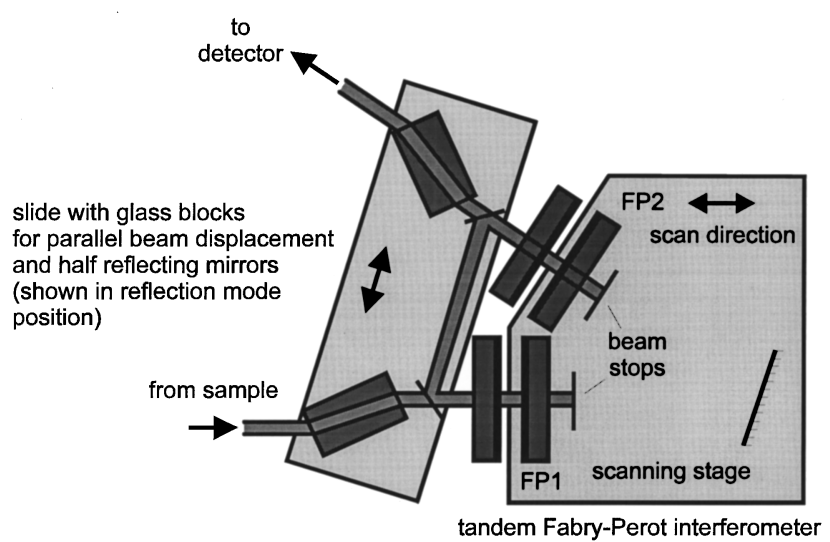

FIG. 2. Schematic view of the optical beam pass in reflection mode. For the change into the transmission mode the slide is moved until the glass blocks and the half reflecting mirrors are outside the beam.

the scanning stage (ramp voltage $V_{Z}$ applied to the $Z$ piezo) two dips in the transmitted light intensity appear as a function of the ramp voltage, i.e., of the mirror spacing, which correspond to the two resonance conditions of the two etalons. Now the parallelism of the etalons can easily be aligned by making the dip as deep as possible by varying $V_{X 1}, V_{Y 1}$ or $V_{X 2}, V_{Y 2}$, respectively.

In order to align the interferometer the optical beam path is first switched into the reflection mode. Each etalon is aligned by optimizing the respective dip of the reflected intensity as a function of the voltage $V_{Z}$ through variation of $V_{X 1}, V_{Y 1}$ and $V_{X 2}, V_{Y 2}$, respectively. This is performed either by the autoalign routine (see Sec. III F below) or manually. For manual alignment we have built a handbox, which is held in the hands of the operator during alignment and connected to the computer. It contains buttons, which can be pressed to modify the settings of $V_{X 1}, V_{Y 1}, V_{X 2}$, $V_{Y 2}, V_{Z}$, and $V_{d Z}$, etc. It will be described in detail in Sec. III B. Since there are only two degrees of freedom for each etalon $\left(V_{X i}, V_{Y i}\right)$ the coarse alignment is easily performed for each etalon. Next the synchronization of both etalons is made by varying $V_{d Z}$ applied to all piezos of FP2 until both dips fall on each other. By varying the offset to the ramp voltage, $V_{Z}$, the dips are moved to the center position of the spectrum. After switching the optical beam path back into the transmission mode, the system is well enough aligned to transmit a sufficient amount of light in the $(3+3)$ path configuration for allowing the active stabilization routines to work.

\section{COMPUTER CONTROL}

\section{A. Overview of the software package}

The developed software package "TFPDAS3" comprises interrupt-driven routines for driving the scanning stage, the active stabilization of the etalon mirrors, routines for the data accumulation and for displaying and handling the data, as well as many functions for controlling the peripheral hardware (acousto-optic modulator, shutters, servo for changing the optic beam pass, automatic search for optimum focus, control of sample positioning, applied magnetic 
field, temperature, etc.). On the computer screen there are displayed the spectrum during accumulation, status information about the interferometer and the performance of the alignment routines, major parameters controlling the performance, and a photon count rate meter.

All peripheral devices can be addressed either by lowlevel commands, like changing the output state of a TTL signal, or by high-level device control routines, like moving or rotating the sample holder to a calibrated value.

TFPDAS3 offers three modes of control: (i) All commands and parameters can be entered through appropriate menus. Parameter editing is performed in separate menus such as for parameters concerning the scan, the sample used, the stabilization, the AOM, the data fit, setup parameters, etc. (ii) All commands and parameters can be entered by one-line commands at a command prompt (so-called "command mode"). (iii) All commands and parameters can be executed from a script file. This allows a very flexible handling of the data accumulation process. Through script control peripheral devices can be controlled, and, therefore, a series of measurements as a function of, e.g., the sample position, the angle of light incidence, or the strength of the magnetic field can be performed. This allows unattended $24 \mathrm{~h}$ operation of the setup. For this purpose we have developed a script language which includes elements like loops, subroutine calls, and input masks for easy parameter configuration. In addition to the parameters discussed above temporary parameters used in script files can be defined.

The basic menu items of TFPDAS3 are (i) data input/ output, (ii) control of the interferometer (alignment, stabilization, data accumulation, diagnostics of all hardware functions, calibration routines), (iii) edit of parameters, (iv) data fitting, (v) the command line mode described above, and (vi) miscellaneous functions. A context sensitive help system provides any information needed by the operator.

In writing the software it has been proven to be very useful to express all frequencies in fractions of the free spectral range (FSR). This unit system has the advantage, that characteristic frequency positions, like the ranges for the drift stabilizer and the finesse optimizer (see below) do not change upon changing the mirror spacing. In a standard measurement a scan is performed between -1.2 and +1.2 FSR thus containing the ghost peaks, i.e., the signals from neighboring transmission orders, at \pm 1 FSR. We usually use 200 channels per FSR. Therefore a scan from -1.2 to 1.2 FSR comprises 480 channels. All these quantities can be modified by editing appropriate parameters in TFPDAS3. In the display of TFPDAS3 the frequency unit can be changed between the units $\mathrm{GHz}, \mathrm{cm}^{-1}, \mu \mathrm{eV}$, and fraction of FSR.

\section{B. Data accumulation and hardware}

The computer interacts with the spectrometer through two plug-in boards, which fill two slots of the PC main board. One board, called the "counter board" hereafter, handles all binary, TTL level input/output. The second board is a commercially available, standard, digital/analog (D/A) board comprising eight analog output lines, which are used to drive the scan piezo $\left(V_{Z}\right)$, the mirror piezos $\left(V_{X 1}, V_{Y 1}\right.$,

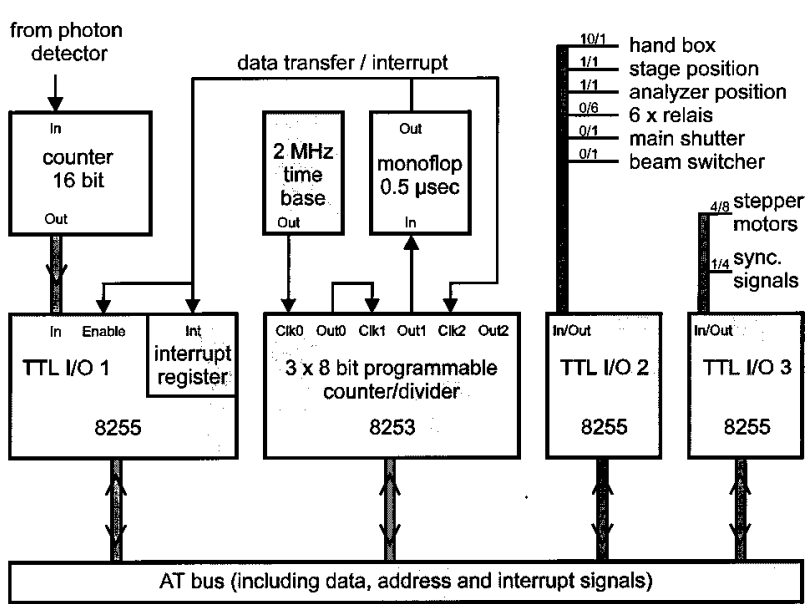

FIG. 3. Schematic view of the counter board. Shown is the complete TTLlevel digital interface hardware used in the setup. The 8255 ICs are programmable $3 \times 8$ bit peripheral interface devices. The 8253 IC is a programmable $3 \times 8$ bit counter and interval timer. The digits at the device connectors of the second and third 8255 IC indicate the numbers of input and output lines for each device.

$\left.V_{X 2}, V_{Y 2}, V_{d Z}\right)$, the acousto-optic modulator, and optionally the power supply for the magnetic field. An optional standard A/D board can be added for reading in analog signals, e.g., for reading the applied magnetic field by a Hall probe.

Data collection is performed by periodically sweeping the stage with a typical time of $0.6 \mathrm{~s}$ per sweep. In this way the time constant of the active stabilization is short enough to allow for a compensation of-mostly thermal driven-drifts in the alignment. The data from the photon detector is stored in two arrays with the array index corresponding to the frequency shift. The first array contains the data of the actual scan, and the second array contains the data accumulated since the start of the measurement.

The central part of the computer control is an interrupt routine, discussed in detail below, which performs the data accumulation and contains the active stabilization routines. The interrupt routine closely interacts with the counter board and the D/A board.

The schematic operation of the counter board is displayed in Fig. 3. A timer generates an interrupt call with a repetition rate which is usually set equal to $1 \mathrm{~ms}$. In the interrupt routine the current content of a counter, counting the pulses from the photon detector, is evaluated and the number of counts collected since the last interrupt event is added to the current channel, the channel index is incremented, and the scan stage is advanced to the next position by incrementing $V_{Z}$. After completion of a sweep the scan stage is set back to the start position in several, so-called retrace steps, which are performed every $10 \mathrm{~ms}$. After the retrace, routines are performed, which generate the correction signals for the active stabilization (see below). The most important routines are the drift stabilizer and the finesse optimizer routines. Next routines are executed, which control the AOM light transmission level, the general operation of the system, like a test for completion of a spectrum (preset number of scans performed), and data manipulation.

The TTL-level pulses generated by the avalanche photon 
detector are counted using a 16 bit counter located on the counter board.

The central element of the counter board is a programmable $3 \times 8$ bit interval timer and counter (8253) used as the time base of the system (see Fig. 3). A $2 \mathrm{MHz}$ quartz and two cascaded 8 bit counters are used to generate the clock signal for calling the interrupt routine. The division ratio is set by software, and it is - as described above-typically 1 $\mathrm{ms}$ during a scan and $10 \mathrm{~ms}$ during a scan retrace. A monoflop triggered by the output of the second counter generates a $0.1-\mu$ s-long trigger signal. This signal is used to enable the transfer of the current value of the photon pulse counter to the first 8255 TTL input/output (I/O) chip. At the same time the trigger signal sets an interrupt flip-flop, which is part of the 8255 chip. The output of the flip-flop register is wired to one of the hardware interrupt lines of the PC (we use Int5, which corresponds to $L P T 2$, not used in our PC). The interrupt routine, triggered by the hardware interrupt, reads out the TTL I/O chip and thereby resets the interrupt register.

The number of counts are obtained by subtracting the previous content of the TTL I/O registers from the new value. In this way a significant dead time caused by resetting the counter each time it is read out is avoided. The currently active channel is incremented by the number of counts, the channel index pointing to the active channel is incremented, and the output voltage, $V_{Z}$, driving the scan piezo, is increased to advance the position of the scanning stage. This procedure is repeated until the sweep is completed.

There is a built-in measure to ensure that no interrupt call is missed by the software. The trigger signal is also wired to the input of a third counter on the 8253 chip. By comparing the content of this counter with an index in the interrupt routine, incremented upon each call of the routine, any missing response of the interrupt routine is easily detected. A diagnostic routine is part of TFPDAS3 which allows a full analysis of the behavior. Using 386 or faster PCs we could not detect any missing interrupt calls. For slower PCs, as we have used in the beginning, we have setup an option, which allows us to dismiss the respective scans in which this problem has occurred.

The counter board provides all TTL-level signals to control peripheral devices. These are lines for switching the scanning stage between the reflection mode and transmission mode position (one output line and one input line from end switches to control completion of move), the position of a polarization analyzer, which can be moved in and out of the light beam (one input and one output line), six auxiliary lines (relais lines), the main shutter to protect the photon detector (one output line), and for switching the light beam in case that a shutter system is used instead of the AOM (one output line).

Using TFPDAS3 the manual alignment is easily performed using a handbox. It has keys to be pressed for variation of the $X$ and $Y$ tilts of the etalon mirrors, the voltages $V_{Z}$ and $V_{d Z}$, the position of the objective, to toggle the optic beam path, and to move in and out the polarization analyzer. Two switches allow to change between the $X$ and $Y$ parameters of FP1 and FP2, and to change the speed of variation between slow and fast. The handbox is connected with 10
TTL-level input lines and one output line, the latter used to enable the handbox and to switch on a light emitting diode (LED) on the handbox as an optic control.

On the counter board a third 8255 chip is used to drive stepper motors for sample and focus lens positioning. For each motor two output signals are generated which are the clock and the direction signal. The state of end switches is monitored through one input line.

We have developed an advanced setup which allows us to map the two-dimensional distribution of spin wave intensities of microwave-excited spin waves with time resolution. It is discussed in detail in a forthcoming article. ${ }^{16}$ In this setup the data accumulation is performed in a separate PC. For synchronization the signals "clock," "scan number is odd/even," "channel index is within a range of interest," and "retrace" are sent to the second PC. An input line "inhibit stab" is used to temporarily disable stabilization, e.g., while the second PC performs a change in sample position.

\section{The drift stabilizer and the finesse optimizer}

Next we discuss the stabilization algorithms. Two different tasks must be performed: (i) The drift stabilizer maintains the frequency alignment between the channel positions and the corresponding transmission frequencies, and (ii) the finesse optimizer performs the mirror alignment routines. Here, there are five independent quantities: the horizontal and vertical parallelism of the first and second etalons with axes $X 1, Y 1$ and $X 2, Y 2$, respectively, and the correlation between the two etalons for maintaining the frequency synchronization. The latter is adjusted by modifying the spacing of FP2 by an amount $d Z$.

For the stabilizer and optimizer routines the signal of the last scan of the central, elastic peak is evaluated and input parameters are calculated. They are the integrated counts over the central peak, $N_{F}$, and the counts of the left and right half of the central peak, $N_{D_{-}}$, and $N_{D+} . N_{F}$ is used as input for the finesse optimizer, and the imbalance of $N_{D-}$ and $N_{D+}$ is used in the drift stabilizer as a measure of the peak shift with respect to its preset zero position. We denote with $C_{0}$ the channel index of zero frequency shift.

The central peak extends over the range of channels $\left[C_{0}-F, C_{0}+F\right]$ with $F$ suitably chosen to contain the peak. The finesse optimizer input, $N_{F}$, is then calculated:

$$
N_{F}=\sum_{i=C_{0}-F}^{C_{0}+F} N(i)
$$

with $N(i)$ the counts in channel $i$. For the drift stabilizer, the range, characterized by the parameter $D$, is evaluated:

$$
\begin{aligned}
& N_{D-}=\sum_{i=C_{0}-D}^{C_{0}} N(i), \\
& N_{D+}=\sum_{i=C_{0}}^{C_{0}+D} N(i) .
\end{aligned}
$$

$F$ and $D$ should be chosen small enough to lie within the central peak, but large enough to cover enough counts for 
obtaining good statistics. We use values of $F$ and $D$ corresponding to frequency shifts of 0.050 and $0.045 \mathrm{FSR}$, respectively.

Drift stabilizer: From the integrated values a correction signal

$$
Z_{D}=c_{D} \times\left(N_{D+}-N_{D^{-}}\right) /\left(N_{D+}+N_{D^{-}}\right)
$$

is calculated. It is a measure of the drift of the central peak with respect to its center position. With the constant $c_{D}$ properly chosen, $Z_{D}$ is applied as a change of offset to the $Z$ scan voltage.

In the finesse optimizer the counts $N_{F}$ are used to generate a correction signal. Since the finesse optimizer routine must maintain stability in five degrees of freedom, 12 scans in pairs of two are used for a full stabilization cycle: Within two consecutive scans the voltage for axis $X_{i}$ is set to $X_{i}$ $-\delta$ in the first scan and to $X_{i}+\delta$ in the second scan. The corresponding finesse signals are $N_{F}(-\delta)$ and $N_{F}(+\delta)$. The correction signal is now calculated using the equation

$$
\begin{gathered}
Z_{F}=c_{F} \times\left[N_{F}(+\delta)-N_{F}(-\delta)\right] / \\
{\left[N_{F}(+\delta)+N_{F}(-\delta)\right] / \delta}
\end{gathered}
$$

with $c_{F}$ a properly chosen parameter. Its value should be chosen large enough to enable a fast convergence, but not too large to avoid oscillations in the convergence.

A full alignment cycle comprises the steps $X 1+\delta, X 1$ $-\delta, Y 1+\delta, Y 1-\delta, d Z+\delta, d Z-\delta, X 2+\delta, X 2-\delta, Y 2$ $+\delta, Y 2-\delta, d Z+\delta$, and $d Z-\delta$. Due to its high sensitivity the $d Z$ alignment steps are performed twice during a full alignment cycle. After each pair of scans a correction signal as in Eq. (5) is calculated and added as an offset to the corresponding parameter. For the two $d Z$ steps we use a separate value for $c_{F}$ for better performance.

For optimum performance the dither amplitude, $\delta$, must be carefully adjusted. Too small a value results in unstable operation, and too large a value reduces the overall transmission. We use a dynamical adjustment of $\delta$, which is described further below.

The values of the range parameters $D$ and $F$ can be set in parameter menus or graphically by moving a cursor on the screen displaying the spectrum.

\section{Control of the acousto-optic modulator and adjustment of the dither amplitude}

For stable operation it is essential to minimize the statistical error in the generated correction signals by using maximum light levels, but avoiding saturation and/or damage of the photon detector. We do so by using an acousto-optic modulator (AOM) mounted between the laser light source and the focusing lens. The light attenuation level is controlled by the software as follows: at four channel positions, denoted $C_{\mathrm{AOM} 1} \ldots C_{\mathrm{AOM} 4}$ hereafter, the transmitted light intensity is toggled between the levels $\mathrm{AOM}_{\text {low }}$ and $\mathrm{AOM}_{\text {high }}$. The value of $\mathrm{AOM}_{\text {high }}$ is usually chosen to $100 \%$ light transmission. The value of $\mathrm{AOM}_{\text {low }}$ can be varied either manually or under software control. The channel positions $C_{\mathrm{AOM} 1}$ and $C_{\mathrm{AOM} 2}$ are used to define the region of the investigated inelastic light signals on the anti-Stokes side of the spectrum, the channel positions $C_{\mathrm{AOM} 3}$ and $C_{\mathrm{AOM} 4}$ that on the Stokes side. During a sweep the light level is switched at $C_{\mathrm{AOM} 1}$ to $\mathrm{AOM}_{\text {high }}$, entering the region, where inelastic signals are studied on the anti-Stokes side of the spectrum. At the channel $C_{\mathrm{AOM} 2}$ the light level is reduced to $\mathrm{AOM}_{\text {low }}$, before the sweep enters the central region of the spectrum, where the strong elastic scattering intensity might overload and/or damage the detector. Consecutively at $C_{\mathrm{AOM} 3}$ the light level set to $\mathrm{AOM}_{\text {high }}$, and at $C_{\mathrm{AOM} 4}$ back to $\mathrm{AOM}_{\text {low }}$. In the standard setting $C_{\mathrm{AOM} 1}, C_{\mathrm{AOM} 2}, C_{\mathrm{AOM} 3}$, and $C_{\mathrm{AOM} 4}$ correspond to $-0.95,-0.05,0.05$, and 0.95 FSR, respectively. The values of $C_{\mathrm{AOM} 1} \ldots C_{\mathrm{AOM} 4}$ can be set in parameter menus or graphically by moving a cursor on the screen displaying the spectrum.

The attenuation factor $\mathrm{AOM}_{\text {low }}$ is dynamically adjusted after each complete finesse loop (12 scans) such that the peak intensity of the central peak (elastic scattering signal), averaged over the finesse loop, is maintained at a preset value (in our setup: 1000000 counts/s) within a given range. This procedure has the enormous advantage that the dynamic range for maintaining stability is increased by the dynamic range of the AOM. In the case of destabilization the system is able to recover by receiving 10 counts in the region $\left[C_{0}\right.$ $\left.-F, C_{0}+F\right]$. By increasing the light level in the central region through the dynamic adjustment of $\mathrm{AOM}_{\text {low }}$ the system is able to recover under nearly all circumstances. If for any circumstance stabilization is lost, the system falls back into the automatic alignment routines (see below) and switches back to data accumulation after successful alignment.

For optimum performance the dither amplitude, $\delta$, should be adjusted carefully. A small value results in high light throughput through the interferometer in case of optimum alignment, but it also yields a high sensitivity to destabilization since then convergence of the active stabilization routines is small. In contrast, a large value is used if a stable operation is needed under rough conditions (e.g., when a noisy device is used close to the setup), but the overall transmission of the spectrometer is smaller.

In our setup the dither amplitude is adjusted automatically as follows: The value of the AOM attenuation level, $\mathrm{AOM}_{\text {low }}$, is used as a monitor for the system stability. If the system is in a destabilizing condition, $\mathrm{AOM}_{\mathrm{low}}$ will increase to allow more light of the central peak region to enter the interferometer in order to compensate the lesser transmission. In this case the value of $\delta$ will be increased until $\mathrm{AOM}_{\text {low }}$ will no further increase. As long as $\mathrm{AOM}_{\text {low }}$ is not increasing the dither amplitude will decrease until either a lower bond value is reached or a balance between the change in $\mathrm{AOM}_{\text {low }}$ and $\delta$ is obtained. In day-to-day use this feature has been proven very useful. In particular when working in a noisy environment with, e.g., running pumps of an ultrahigh vacuum (UHV) system or of a cryostat apparatus, the automatic adjustment of the dither amplitude guarantees stable operation. In TFPDAS3 we have included an option which disables data accumulation while the dither amplitude is not at minimum. This option guarantees optimum light transmission through the interferometer. This option is needed if 
quantitative measurements of the light scattering cross section are performed.

$C_{\mathrm{AOM} 1} \ldots C_{\mathrm{AOM} 4}$ are the boundaries of the regions, where the inelastic light scattering signals are analyzed. To increase the ratio of time the spectrometer spends in these regions with respect to the total scan time, the scan speed can be reduced by a preset factor in these regions. If only a very small part of the spectrum is investigated the time needed for the measurement is largely reduced by setting $C_{\mathrm{AOM} 1} \ldots C_{\mathrm{AOM} 4}$ very close to the region and by reducing the scan speed in this region.

\section{E. The main shutter}

A shutter, called main shutter, is mounted in front of the photon detector. It is controlled by the software. The shutter is opened upon starting a measurement and closed after completion of the measurement. This shutter is particularly needed, if a photomultiplier is used as the detector. Photomultiplier tubes are very sensitive to overload with a potential of damage. To avoid any damage the current count rate is monitored within the interrupt routine. Within a response time of $1 \mathrm{~ms}$ the main shutter is closed if the count rate exceeds a preset value. The response time is sufficiently small to protect the photomultiplier even if it is exposed erroneously to daylight. The software solution makes separate protective measures unnecessary.

\section{F. The autoalignment routine}

One of the strengths of our software is the implementation of the autoalignment routine for the coarse alignment of the interferometer. In the active stabilization routines described above six parameters are constantly optimized. It is clear, that after power-on the settings for mirror tilts, scan offset and mirror correlation are too far away from alignment to allow enough photons to pass through the interferometer and to provide a signal strong enough for the stabilization routines to optimize on.

The manual coarse alignment has been described in Sec. II B. The autoalignment routine works very similar. It can, however, perform the necessary steps much faster. During the alignment the system operates in a fast scanning mode with 128 channels per scan for fast convergence. While scanning in the reflection mode the AOM level $\mathrm{AOM}_{\text {low }}$ is adjusted after each scan to keep the average count rate at a preset value. The value of $\mathrm{AOM}_{\text {low }}$ might serve as a measure of light intensity scattered elastically from the sample.

First the servo controlling the optic beam pass switches the system into the reflection mode position and all etalon piezo voltages are reset to their default values. The voltages applied to the piezos of the second etalon are then set to their limiting values. In this way FP2 is detuned in a maximum way and no significant dips in the alignment sweep appear from this etalon. The system is scanning over a range of 1.2 FSR.

In the first coarse aligning step the two piezo voltages $V_{X 1}$ and $V_{Y 1}$ of FP1 are systematically varied by walking their values in the $X 1-Y 1$ parameter plane in a spiral-like fashion starting at the center, until a sufficiently deep dip in the signal is found. We use a reflection value of $40 \%$ to identify a dip in this step. Next the piezo voltage values of the first etalon are stored, the first etalon is detuned, the second etalon is reset and the coarse alignment routine is applied to the second etalon mirror. After restoring the stored optimized values of FP1, each etalon is now coarsely aligned. Next both dips are moved to preset values (0.4 FSR apart) by adjusting $V_{d Z}$ and the offset to $V_{Z}$. Using a regulafalsi algorithm the voltage values $V_{X 1}, V_{Y 1}$ and $V_{X 2}, V_{Y 2}$ are systematically varied, until the reflectivity in the minimum of the dips is sufficiently small ( $<5 \%$ in our setup). Finally the $Z$ and $d Z$ parameters are adjusted in order to synchronize the two etalons and to set the position of the central peak.

After completion of these alignment steps the optical beam path is switched to the transmission mode. In the fast scanning mode the drift stabilizer and the finesse optimizer are used to converge towards optimum alignment.

It should be noted, however, that for a good performance a manual prealignment of the system, by adjusting mechanically the etalon mirrors (by rotating the mechanic micrometer screws on the mirror mounts) and the scan piezo, is needed. A good manual prealignment is achieved when the parameters for optimum alignment lie roughly in the middle of their respective adjustment ranges. We need to perform prealignment about every four weeks. There is no need to redo the prealignment in case of a change of the free spectral range. If the prealignment of the system is good enough the system is ready for starting measurements within a fraction of a minute: First the reflection mode is enabled and the system is aligned. Second the optic beam pass is switched to multipass operation, and in the fast scanning transmission mode an optimum alignment is achieved and measurements can be started. This all takes place by a single key stroke in TFPDAS3.

\section{G. The autofocus routine}

If the system is taking a series of spectra as a function of various external parameters, like the lateral focus position on the sample or the strength of an applied field, maintaining a good focus is essential for stable operation. In our system the alignment of the distance between the objective lens and the sample can be performed automatically. In the reflection mode the detected maximum light intensity (outside from dips) is a good measure of the light intensity reflected from the sample. We found that in most cases an optimum focus position is obtained if the light intensity is found to be at maximum. In the alignment routine the objective lens is first moved to a preset position, which is a bit closer than the anticipated optimum position. Then scanning of the system is started, the maximum light intensity is measured and the objective lens is slowly moved away from the sample. The $\mathrm{AOM}$ level $\mathrm{AOM}_{\text {low }}$ is constantly adjusted to protect the photon detector from overload; the value of $\mathrm{AOM}_{\text {low }}$ is used as a measure of the light intensity. The light intensity as a function of the position of the objective lens is evaluated and the maximum position is determined by a peak finding routine. Finally the objective lens is moved to the position 
where the maximum light intensity was found.

The procedure works generally fine for metallic surfaces with not too many surface defects. However, by its nature, it does not work for transparent samples, in particular if the laser focus is supposed to lie inside the sample.

\section{H. The fitting routines}

The system contains routines to fit the elastic and inelastic peaks in the spectra to determine their frequency positions, heights, and widths. The frequency position of an excitation can be determined with an accuracy much better than the experimental resolution by evaluating the full peak structure. By involving fitting routines an increase in the data accuracy is obtained, provided that experimental uncertainties, like a scan nonlinearity or any offset in the scan, is below the error of the fit and the peak shape is not asymmetric. For the fitting profiles Lorentzian and Gaussian line shapes and powers thereof are used as well as background subtraction. A coarse estimate of the parameters used as start parameters for the fit is obtained by a peak finding algorithm. To obtain reliable fits we perform a full error analysis of the fit results including an investigation of the parameter correlation. Details of the fit routines will not be discussed here, since, in general, many fit algorithms are available for this purpose.

The data fit has two purposes. First it is used to obtain accurate values for the peak positions, peak heights and peak widths. Second, fits are used to achieve a postlinearization of the scan as follows. A (mostly) linear scan is obtained by the hardware feedback loop using a capacitive distance detector for the etalon mirror plates, as discussed in Sec. II A. A residual nonlinearity will have its largest contribution in its lowest order, which is quadratic. To correct these quadraticorder nonlinearities the exact frequency position of three channels must be known. The positions of the central peak $(=0)$ and the two ghost peaks $( \pm \mathrm{FSR})$ will serve for this purpose, since the position of the ghost peaks are known from the mirror spacing, which is measured with high accuracy. All three positions are usually contained in the spectrum, since the scan range is usually chosen between -1.2 and +1.2 FSR. By fitting the positions of these three peaks in the spectra the coefficients of a quadratic correction are derived which are then used to recalibrate the frequency scale. Normally the recalibration procedure yields only very small corrections. However it has been proven very useful to monitor the correction factors on a regular basis, since any misoperation of the system results in largely modified correction factors.

\section{Miscellaneous routines}

A number of routines serve for various additional purposes. Diagnostic screens allow us to monitor and manipulate the low-level signals sent to and received from all peripheral devices, such as all TTL-level lines and the analog input and output signals. A second diagnostic screen allows us to test the counting board, to monitor the photon detector and to measure the dark count rate with high accuracy. A service screen displays the transmitted light intensity in re-

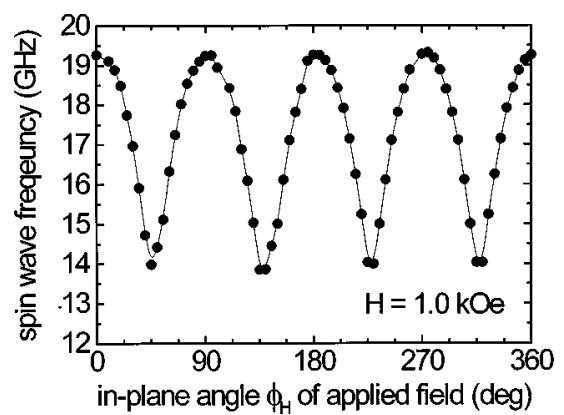

FIG. 4. Measured spin wave frequencies for a 30-nm-thick $\mathrm{Fe}(001)$ film as a function of the in-plane angle of the applied field, $\phi_{H}$. For protection against oxidation the film is covered by a $\mathrm{Cr}$ cap layer.

flection mode, which is used for manual adjustments in the prealignment of the interferometer, e.g., if the light path through the second pin hole or the focus on the photon detector is adjusted.

For testing and adjusting the mechanical damping of the translation stage, a service routine is built-in, in which a square wave signal with variable frequency and amplitude is applied to the scan piezo. The mechanical ringing of the translation stage caused by the sudden change in position is measured using an oscilloscope connected to the $Z$-feedback signal of the stage.

Crucial parameters in the software are the calibration factors which translate the numerical values in mechanical translations of the scanning stage $(Z)$ or the second etalon $(d Z)$. A service routine is builtin for adjusting these parameters. In the case of the calibration factor for the scanning stage the adjustment is fully automatic: After performing a few test scans the position of the ghost peaks are determined by a fit and compared with the nominal positions. From the difference the correction for the calibration factor is calculated.

A number of routines allows data handling like storing, loading and deleting spectra, and smoothing of spectra. Together with each spectrum all parameters relevant to the spectrum are stored. An interface routine exists for exporting data to standard data analysis and graphics software.

For communication with supplementary equipment the serial port is fully supported by script commands.

\section{EXAMPLES}

We will illustrate the performance of our setup by three examples. The first two concern spin wave excitations, and the reader is referred to the original literature for more information concerning the physical background. ${ }^{17,18,20-28}$ For a recent review see Ref. 29.

Figure 4 shows the measured spin wave frequencies in a 30-nm-thick epitaxial $\mathrm{Fe}(001)$ film. The films were deposited onto $5 \mathrm{~mm} \times 10 \mathrm{~mm} \mathrm{GaAs}(001)$ substrates, covered by a 150 nm-thick face-centered-cubic (fcc) $\mathrm{Ag}(001)$-buffer layer. A Cr cap layer has been deposited onto the Fe film to avoid oxidation. The sample preparation was performed in a UHV system with a base pressure better than $10^{-10}$ mbar by means of molecular beam epitaxy. The chemical and crystallographic analysis of the surfaces has been performed by in 


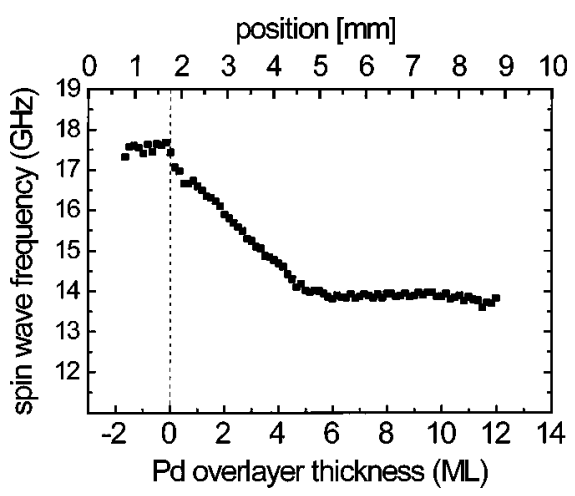

FIG. 5. Spin wave frequency for a Pd wedge on a 15-ML-thick Fe film, measured at $H=1 \mathrm{kOe}$, as a function of $d_{\mathrm{Pd}} . P_{\mathrm{Pd}}<0$ corresponds to the uncovered Fe film and is used as a reference.

situ Auger electron spectroscopy (AES) and low energy electron diffraction (LEED). The growth procedure is reported in more detail in Ref. 26.

In Fig. 4 the in-plane angle $\phi_{H}$ of the external field, measured with respect to the in-plane [100] direction, is varied by rotating the sample about the surface normal, thus maintaining the perpendicular orientation between external field and the scattering plane. The spin wave frequencies are measured at every angular increment of $5^{\circ}$. From the obtained data the in-plane fourfold anisotropy is deduced. ${ }^{26}$

Of particular interest is the modification of surface anisotropies by an overlayer. We used Pd, which was deposited onto the Fe film. The best Pd layers were grown on $\mathrm{Fe}(001)$ at a growth temperature of $300{ }^{\circ} \mathrm{C}$ with a growth rate of 0.02 monolayers (ML) per second. Good coverage of the $\mathrm{Fe}$ layers by $\mathrm{Pd}$ at $300{ }^{\circ} \mathrm{C}$ is attested by the exponential decay of the Fe Auger peak $(47 \mathrm{eV})$ intensity with increasing $\mathrm{Pd}$ thickness. The Fe films are nearly completely covered by $\mathrm{Pd}$ at $d_{\mathrm{Pd}} \approx 2 \mathrm{ML}$.

To check the influence of the Pd overlayer it is convenient to grow the Pd layer as a wedge. We have studied such a sample with the Pd thickness varying across the sample between 0 and $10 \mathrm{ML}$. The thickness dependence of the spin wave frequency is then easily determined by scanning the light beam across the sample. The resulting spin wave frequencies are shown in Fig. 5. It is clearly seen, that the Pd deposition dramatically changes the spin wave frequencies even after the $\mathrm{Fe}$ surface is completely covered by the Pd layers for a Pd thickness larger than 2 ML. From the data in Fig. 5 the surface anisotropies as a function of $d_{\mathrm{Pd}}$ are derived. For a full report see Ref. 26.

We have developed an advanced version of our setup, which allows us to map the two-dimensional distribution of spin waves in a magnetic film. ${ }^{17,18}$ The spin waves are excited by a microwave antenna and propagate away from the antenna. Applying high microwave fields nonlinear processes like self-focusing are observed. ${ }^{17}$ We also have developed a method to yield time resolution: By exciting spin wave pulses by microwave pulses applied to the antenna, the time between the launch of the pulse and the detection of the signal by the photon detector can be analyzed. ${ }^{18}$ Using time and space resolved Brillouin light scattering, we were able to detect the propagation of so-called spin wave bullets in

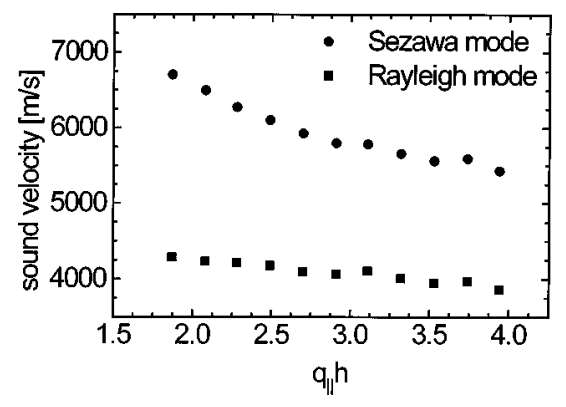

FIG. 6. Measured dispersion curve for the Rayleigh and the first Sezawa mode of a hexagonal BN film of thickness $h=170 \mathrm{~nm}$ deposited on $\mathrm{Si}(001)$.

yttrium-iron-garnet films. A spin wave bullet is an excitation which does not change its shape in both in-plane directions and in time, and which is stabilized by dissipation. ${ }^{18}$ The system will be described in a forthcoming publication. ${ }^{16}$

Apart from investigations of magnetic systems, which are the main area of our interest, elastic properties are investigated by Brillouin light scattering very often. As an example Fig. 6 shows the measured sound velocities of a hexagonal-phase boron nitride (BN) film. ${ }^{30}$ The film was deposited at $620 \mathrm{~K}$ by unbalanced magnetron sputtering from a hexagonal BN target onto a $\mathrm{Si}(001)$ substrate with simultaneous $\mathrm{Ar}$ ion plating. The substrate was ion etched for 10 min using Ar with an ion energy of $300 \mathrm{eV}$. The film thickness is $170 \mathrm{~nm}$ and the mass density is $2.14 \mathrm{~g} / \mathrm{cm}^{3}$. The obtained dispersion curve shown in Fig. 6 shows a very smooth variation of the measured sound velocity as a function of the product of the in-plane wavevector component, $q_{\|}$, and the film thickness $h$.

\section{DISCUSSION}

We have developed a computer control for a Sandercock-type multipath tandem Fabry-Perot interferometer. The system offers ultimate versatility, stability, and user friendliness, and it extends the applicability of tandem Fabry-Perot spectroscopy to new areas, which in the past were not easily accessible. It can be operated by any scientist with a few days instruction about the operation of the spectrometer. The setup has now been used in our laboratory by many diploma and Ph.D. students with their primary interest in the materials they are studying. However, we would like to note, that although the system is very easy to use in every day operation, a person senior in the Brillouin light scattering technique is still needed in the laboratory for occasional trouble shooting and performing the prealignment procedures, as is the case for any high-level optical system.

The achieved advancements described in this report have helped us to explore many new and exciting areas in modern surface physics and in magnetism. We believe, that the potential of Brillouin light scattering will continue to grow steadily in the future.

\section{ACKNOWLEDGMENTS}

The author would like to thank J. R. Sandercock for many stimulating discussions, and (in chronological order) K. Kylmanen, V. Harzer, P. Krams, J. Fassbender, C. 
Mathieu, M. Bauer, and J. Jorzick for the input they provided while working with the system, and the Deutsche Forschungsgemeinschaft for financial support.

${ }^{1}$ J. R. Sandercock, Solid State Commun. 26, 547 (1978).

${ }^{2}$ J. R. Sandercock, Light Scattering from Thermally Excited Surface Phonons and Magnons, Proceedings of the 7th International Conference on Raman Spectroscopy, edited by W. F. Murphy (North-Holland, Amsterdam, 1980), p. 364.

${ }^{3}$ J. R. Sandercock, Trends in Brillouin Scattering: Studies of Opaque Materials, Supported Films, and Central Modes, Topics in Applied Physics, Vol. 51 (Springer, Berlin, 1982), p. 173.

${ }^{4}$ R. Mock, B. Hillebrands, and J. R. Sandercock, J. Phys. E 20, 656 (1987).

${ }^{5}$ A. Yoshihara, Jpn. J. Appl. Phys., Part 1 33, 3100 (1994).

${ }^{6}$ J. Dil, N. C. J. A. van Hijningen, F. van Dorst, and R. M. Aarts, Appl. Opt. 20, 1374 (1981).

${ }^{7}$ P. Krams, F. Lauks, R. L. Stamps, B. Hillebrands, and G. Güntherodt, Phys. Rev. Lett. 69, 3674 (1992).

${ }^{8}$ J. R. Sandercock, Proc. SPIE 732, 157 (1987).

${ }^{9}$ J. R. Sandercock, Proceedings of the 2nd International Conference on Light Scattering in Solids, edited by M. Balkanski (Flammarion, Paris, 1971), p. 9

${ }^{10}$ S. M. Lindsay and I. W. Shepherd, Rev. Sci. Instrum. 48, 1228 (1977).

${ }^{11}$ S. M. Lindsay, M. W. Anderson, and J. R. Sandercock, Rev. Sci. Instrum. 52, 1478 (1981).

${ }^{12}$ D. Bechtle, Rev. Sci. Instrum. 47, 1377 (1976).

${ }^{13}$ K. Weishaupt, S. H. Anders, R. G. Ebele, and M. Pietralla, Rev. Sci. Instrum. 68, 3996 (1997).

${ }^{14}$ J. A. Bell, Dissertation, University of Arizona, 1987.

${ }^{15}$ T. Blachowicz, R. Bukowski, and Z. Kleszczewski, Rev. Sci. Instrum. 67, 4057 (1996).
${ }^{16} \mathrm{M}$. Bauer, O. Büttner, and B. Hillebrands (unpublished).

${ }^{17}$ M. Bauer, C. Mathieu, S. O. Demokritov, P. A. Kolodin, S. Sure, H. Dötsch, A. N. Slavin, and B. Hillebrands, Phys. Rev. B 56, R8483 (1997).

${ }^{18}$ M. Bauer, O. Büttner, S. O. Demokritov, B. Hillebrands, Y. Grimalsky, Yu. Rapoport, and A. N. Slavin, Phys. Rev. Lett. 81, 3769 (1998).

${ }^{19}$ Dr. J. R. Sandercock, Lindmoosstraße 10, 8910 Affolten a.A., Switzerland.

${ }^{20}$ C. Mathieu, J. Jorzick, A. Frank, S. O. Demokritov, B. Hillebrands, A. N. Slavin, B. Bartenlian, C. Chappert, D. Decanini, F. Rousseaux, and E. Cambril, Phys. Rev. Lett. 81, 3968 (1998).

${ }^{21}$ J. Fassbender, G. Güntherodt, C. Mathieu, B. Hillebrands, R. Jungblut, J. Kohlhepp, M. T. Johnson, D. J. Roberts, and G. A. Gehring, Phys. Rev. B 57, 5870 (1998).

${ }^{22}$ C. Mathieu, M. Bauer, B. Hillebrands, J. Fassbender, G. Güntherodt, R. Jungblut, J. Kohlhepp, and A. Reinders, J. Appl. Phys. 83, 2863 (1998).

${ }^{23}$ C. Hartmann, C. Mathieu, S. Kim, I. Schuller, and B. Hillebrands, Phys. Rev. B 55, 14074 (1997).

${ }^{24}$ C. Mathieu et al., Appl. Phys. Lett. 70, 2912 (1997).

${ }^{25}$ B. Hillebrands, C. Mathieu, M. Bauer, S. O. Demokritov, B. Bartenlian, C. Chappert, D. Decanini, F. Rousseaux, and F. Carcenac, J. Appl. Phys. 81, 4993 (1997).

${ }^{26}$ S. O. Demokritov, C. Mathieu, M. Bauer, S. Riedling, O. Büttner, H. de Gronckel, and B. Hillebrands, J. Appl. Phys. 81, 4466 (1997).

${ }^{27}$ B. Hillebrands, J. Fassbender, P. Krams, C. Mathieu, G. Güntherodt, and R. Jungblut, Il Vuoto, Scienza e Tecnologia 25, 24 (1997).

${ }^{28}$ B. Hillebrands, J. Fassbender, R. M. Jungblut, G. Güntherodt, D. J. Roberts, and G. A. Gehring, Phys. Rev. B 53, 10548 (1996).

${ }^{29}$ B. Hillebrands, Brillouin Light Scattering from Layered Magnetic Structures, in Light Scattering in Solids VII, edited by G. Güntherodt and M. Cardona (Springer, Heidelberg, 1999).

${ }^{30}$ T. Wittkowski, P. Cortina, V. Wiehn, J. Jorzick, K. Jung, and B. Hillebrands (unpublished). 